TTP95-14, hep-ph/9507420

\title{
Electroweak Radiative Corrections To Polarized Møller Scattering Asymmetries
}

\author{
Andrzej Czarnecki \\ Institut für Theoretische Teilchenphysik, Universität Karlsruhe, \\ D-76128 Karlsruhe, Germany \\ William J. Marciano \\ Physics Department, Brookhaven National Laboratory, \\ Upton, New York 11973 \\ and \\ Institute for Nuclear Theory, Box 351550, \\ University of Washington, Seattle, WA 98195-1550
}

\begin{abstract}
One loop electroweak radiative corrections to left-right parity violating Møller scattering $\left(e^{-} e^{-} \rightarrow e^{-} e^{-}\right)$asymmetries are presented. They reduce the standard model (tree level) prediction by $40 \pm 3 \%$ where the main shift and uncertainty stem from hadronic vacuum polarization loops. A similar reduction also occurs for the electron-electron atomic parity violating interaction. That effect can be attributed to an increase of $\sin ^{2} \theta_{W}\left(q^{2}\right)$ by $3 \%$ in running from $q^{2}=m_{Z}^{2}$ to 0 . The sensitivity of the asymmetry to "new physics" is also discussed.
\end{abstract}

\section{INTRODUCTION}

The chiral structure of the standard $S U(2)_{L} \times U(1)_{Y}$ model implies a predictable degree of parity violation in all physical processes, ranging from low energy atomic phenomena to high energy $Z$ boson production asymmetries. Precision experimental studies of those predictions test the standard model at the tree and quantum loop level. A deviation from expectations would point to "new physics".

One interesting class of parity violation measurements involves the scattering of longitudinally polarized (left or right-handed) electrons on an unpolarized target. The left-right scattering asymmetry

$$
A_{L R} \equiv \frac{\mathrm{d} \sigma_{L}-\mathrm{d} \sigma_{R}}{\mathrm{~d} \sigma_{L}+\mathrm{d} \sigma_{R}}
$$


is manifestly parity violating and measures the interference between electromagnetic and weak neutral current amplitudes. A classic example is the now famous SLAC asymmetry measurement for deep-inelastic polarized $e-D$ scattering [1]. That study confirmed the standard model's weak neutral current structure and provided a good determination of the weak mixing angle, $\sin ^{2} \theta_{W}$ (to about $\pm 10 \%$ ). One could envision pushing such asymmetry measurements to much higher levels of precision. Indeed, a later measurement of elastic polarized $e-C$ scattering [2] indicated that systematic uncertainties as small as $10^{-8}$ were achievable in asymmetry experiments.

Given the possibility of very high precision asymmetry measurements using present day facilities and technology, it is interesting to investigate what one can learn from such experiments. In that spirit, we consider here the case of polarized Møller scattering $e^{-} e^{-} \rightarrow e^{-} e^{-}$. Our primary focus will be on the use of a very intense highly polarized $\left(P_{e}>0.8\right)$ electron beam in fixed target unpolarized electron scattering.

The tree level prediction for that asymmetry was examined a number of years ago [3]. The interference between electromagnetic and weak neutral current amplitudes in fig. 1 gives rise to the standard model prediction

$$
\begin{aligned}
& A_{L R}\left(e^{-} e^{-} \rightarrow e^{-} e^{-}\right) \\
& \quad=\frac{G_{\mu} Q^{2}}{\sqrt{2} \pi \alpha} \frac{1-y}{1+y^{4}+(1-y)^{4}}\left(1-4 \sin ^{2} \theta_{W}\right)
\end{aligned}
$$

where

$$
\begin{aligned}
G_{\mu} & =1.16639(1) \times 10^{-5} \mathrm{GeV}^{-2} \\
\alpha^{-1} & =137.036 \\
Q^{2} & =-q^{2} \equiv y\left(p^{\prime}+p\right)^{2}=y\left(2 m_{e}^{2}+2 m_{e} E_{\text {beam }}\right)_{\text {fixed target }} \\
q^{2} & =\left(p^{\prime}-p\right)^{2}
\end{aligned}
$$

and the weak mixing angle is roughly $\sin ^{2} \theta_{W} \approx 0.23$. In that expression, terms of order $m_{e} / E_{\text {beam }}$ and $m_{e} / Q$ have been dropped, since we assume $m_{e}^{2} \ll Q^{2} \ll m_{Z}^{2}$.

For fixed target experiments, the asymmetry in (2) is very small because of the tiny $G_{\mu} Q^{2}$ factor and (to a lesser extent) the $1-4 \sin ^{2} \theta_{W}$ suppression factor. Employing a $Z$ pole value, $\sin ^{2} \theta_{W}=0.2314$, and choosing $y=1 / 2$ where the asymmetry is maximal, one finds (for $100 \%$ beam polarization, $P_{e}=1$ ) the tree level prediction

$$
A_{L R}\left(e^{-} e^{-} \rightarrow e^{-} e^{-}\right) \approx 6 \times 10^{-9}\left(E_{\text {beam }} / 1 \mathrm{GeV}\right)
$$

That small an asymmetry may at first sight appear impossible to measure. An experimental group has, however, taken up the challenge and studied the possibility of such a measurement [4. They envision using the SLAC $50 \mathrm{GeV}$ beam (such that $A_{L R}^{\text {tree }} \approx 3 \times 10^{-7}$ ) and operating with very high, well monitored polarization $\left|P_{e}\right| \gtrsim 0.8$. They estimate that using a thick hydrogen target, a statistical precision of $\pm 10^{-8}$ in $A_{L R}$ is achievable in a 3 month run. That corresponds to an accuracy of $\pm 3 \%$ of the standard model tree level prediction and a determination of $\sin ^{2} \theta_{W}$ to \pm 0.0006 . Keeping systematic uncertainties at or below that level is difficult, but its technical feasibility has been experimentally demonstrated. Indeed, 
the experimental feasability study suggests that a measurement of $A_{L R}$ with a total error of $\pm 1.4 \times 10^{-8}$ is possible.

The number of scattering events required for a $10^{-8}$ statistical accuracy is very large, $\sim 10^{16}$. However, such a large data set requirement is not so daunting when one considers the gigantic cross-section in Møller scattering at low $Q^{2}$. (A realistic experiment at SLAC would have $\left\langle Q^{2}\right\rangle \approx 0.02 \mathrm{GeV}^{2}$.)

A measurement of $\Delta A_{L R}$ to $\pm 1.4 \times 10^{-8}$ is only useful if one knows the standard model prediction to that level of certainty. Such precision requires the inclusion of quantum loop effects. Indeed, because the tree level prediction is suppressed by $1-4 \sin ^{2} \theta_{W}$, one anticipates that the relative size of one loop contributions without such a suppression factor will be quite big and that indeed turns out to be the case. In section III, we present the complete one loop radiative corrections to $A_{L R}$ and show that they reduce the standard model prediction by about $40 \%$. That reduction results mainly from $\gamma-Z$ mixing via hadronic vacuum polarization effects. Hadronic loops necessarily entail theoretical uncertainty. However, we show that the uncertainty is conservatively at the $\pm 10^{-8}$ level in the experiment under discussion and thus well matched to envisioned experimental errors. We describe how the theoretical uncertainty could be further reduced by future studies. We also show how the reduction in $A_{L R}$ can be viewed as the running of $\sin ^{2} \theta_{W}\left(q^{2}\right)$ as $q^{2}$ varies from $m_{Z}^{2}$ to $\left|q^{2}\right| \approx 0.02 \mathrm{GeV}^{2}$ which is of relevance for Møller scattering in the planned fixed target experiment.

As a byproduct of our study, we also show that the electron-electron parity violating neutral current interaction is similarly reduced by about $40 \%$ with respect to tree level expectations.

Given the possibility of measuring $\Delta A_{L R}$ to $\pm 1.4 \times 10^{-8}$, one can also ask what "new physics" would be probed? Also, how does such a measurement compare with other precision studies, such as Atomic Parity Violation which has already reached the 1-2\% level and where further improvement is anticipated? To illustrate the utility of polarized $e^{-} e^{-}$scattering, we examine in Section III several "new physics" scenarios such as effects of $Z^{\prime}$ bosons, S, T, U, $\mathrm{V}, \mathrm{W}$ and $\mathrm{X}$ loop effects, and constraints on an anomalous electron anapole moment. The potential of a $\pm 1.4 \times 10^{-8}$ measurement of $A_{L R}$ is compared with various other precision electroweak experiments, particularly atomic parity violation.

In Section $\mathbb{\square}$, we summarize our conclusions and comment on possible future expectations.

\section{ONE LOOP ELECTROWEAK RADIATIVE CORRECTIONS}

Specification of the one loop radiative corrections to $A_{L R}\left(e^{-} e^{-}\right)$requires that we properly define the renormalized parameters that are used in the tree level expression. Our

prescription is fairly conventional. We choose $G_{\mu}$ defined by the muon lifetime formula [5, 6]

$$
\begin{aligned}
\tau_{\mu}^{-1}=\frac{G_{\mu}^{2} m_{\mu}^{5}}{192 \pi^{3}} f\left(\frac{m_{e}^{2}}{m_{\mu}^{2}}\right)\left(1+\frac{3}{5} \frac{m_{\mu}^{2}}{m_{W}^{2}}\right) \\
\times\left[1+\frac{\alpha\left(m_{\mu}\right)}{2 \pi}\left(\frac{25}{4}-\pi^{2}\right)\right]
\end{aligned}
$$




$$
\begin{aligned}
f(x) & \equiv 1-8 x+8 x^{3}-x^{4}-12 x^{2} \ln x, \\
\alpha\left(m_{\mu}\right) & \approx 1 / 136
\end{aligned}
$$

That definition leads to the value of $G_{\mu}$ in (3). Of course, many of the loop corrections to muon decay have been absorbed into $G_{\mu}$. Those corrections are needed when we express neutral current amplitudes in terms of $G_{\mu}$ and will give rise to part of the radiative corrections to $A_{L R}$. Fortunately, those effects are known from previous studies $[7-9$.

The fine structure constant $\alpha$ in (2) is defined by Thomson scattering at $q^{2}=0$ and found to have the value in (3). That quantity is a holdover from atomic physics studies and not always appropriate as a weak loop expansion parameter. For that reason, we prefer to employ $\alpha\left(m_{Z}\right)$

$$
\alpha^{-1}\left(m_{Z}\right)=127.9 \pm 0.1
$$

defined by $\overline{\mathrm{MS}}$ (modified minimal subtraction) at $\mu=m_{Z}$ in short distance dominated loop corrections. By that judicious choice, we avoid inducing 2 loop effects that would be $\sim 7 \%$ of the one loop corrections. Note, however, that some of the most important loop corrections (in particular $\gamma Z$ mixing loops) are better (and more appropriately) parametrized by $\alpha$ [10].

The renormalized weak mixing angle will be defined by $\overline{\mathrm{MS}}$ at scale $\mu=m_{Z}$, $\sin ^{2} \theta_{W}\left(m_{Z}\right)_{\overline{\mathrm{MS}}}$. The use of that scheme simplifies the form of the radiative corrections. For readers more comfortable with $\sin ^{2} \theta_{W}^{\text {eff }}$ used in LEP and SLC asymmetries, there is a simple numerical translation [11]

$$
\sin ^{2} \theta_{W}\left(m_{Z}\right) \overline{\mathrm{MS}}=\sin ^{2} \theta_{W}^{\mathrm{eff}}-0.0003
$$

The analytic form of the radiative corrections in that translation is extremely complicated and will not be given here.

For input, we use

$$
\sin ^{2} \theta_{W}\left(m_{Z}\right) \overline{\mathrm{MS}}=0.2314
$$

which is consistent with $Z$ pole measurements as well as the indirect determinations that use $\alpha, G_{\mu}$ and $m_{Z}=91.190 \mathrm{GeV}$ along with

$$
\begin{aligned}
m_{t}\left(m_{t}\right)_{\overline{\mathrm{MS}}} & \equiv m_{t}=170 \mathrm{GeV} \\
m_{H} & =(\text { Higgs Mass })=200 \mathrm{GeV}
\end{aligned}
$$

That input requires for standard model consistency, $m_{W}=80.39 \mathrm{GeV}$, a value we also adhere to.

Given the above renormalization prescription, we can now unambiguously write down the one loop radiative corrections to $A_{L R}\left(e^{-} e^{-}\right)$. Some parts can be obtained from existing calculations while others require a new study. In total, we find (2) is modified as follows

$$
\begin{aligned}
A_{L R}\left(e^{-} e^{-}\right) & =\frac{\rho G_{\mu} Q^{2}}{\sqrt{2} \pi \alpha} \frac{1-y}{1+y^{4}+(1-y)^{4}} \\
\times\{1 & -4 \kappa(0) \sin ^{2} \theta_{W}\left(m_{Z}\right) \overline{\mathrm{MS}}+\frac{\alpha\left(m_{Z}\right)}{4 \pi s^{2}} \\
& -\frac{3 \alpha\left(m_{Z}\right)}{32 \pi s^{2} c^{2}}\left(1-4 s^{2}\right)\left[1+\left(1-4 s^{2}\right)^{2}\right] \\
& \left.+F_{1}\left(y, Q^{2}\right)+F_{2}\left(y, Q^{2}\right)\right\}
\end{aligned}
$$


where

$$
\begin{aligned}
& s \equiv \sin \theta_{W}\left(m_{Z}\right) \overline{\mathrm{MS}} \\
& c \equiv \cos \theta_{W}\left(m_{Z}\right) \overline{\mathrm{MS}}
\end{aligned}
$$

The quantity $\rho=1+\mathcal{O}(\alpha)$ comes about because we have chosen to normalize the weak neutral current amplitude in terms of the muon decay constant $G_{\mu}$. From earlier work [8], one finds that the renormalization of $G_{\mu}$ combined with vertex and self-energy renormalizations of the $Z$ amplitude gives

$$
\begin{aligned}
& \rho=1+\frac{\alpha\left(m_{Z}\right)}{4 \pi}\left\{\frac{3}{4 s^{4}} \ln c^{2}-\frac{7}{4 s^{2}}+\frac{3}{4 s^{2}} \frac{m_{t}^{2}\left(m_{t}\right) \overline{\mathrm{MS}}}{m_{W}^{2}}\right. \\
& \left.+\frac{3}{4} \frac{\xi}{s^{2}}\left(\frac{\ln \left(c^{2} / \xi\right)}{c^{2}-\xi}+\frac{1}{c^{2}} \frac{\ln \xi}{1-\xi}\right)\right\} \\
& \xi=m_{H}^{2} / m_{Z}^{2}
\end{aligned}
$$

Numerically, for a Higgs mass, $m_{H}=200 \mathrm{GeV}$, and top mass $m_{t}\left(m_{t}\right) \overline{\mathrm{MS}}=170 \mathrm{GeV}$, one finds

$$
\rho=1.00122
$$

The smallness of that correction is due to accidental cancellations.

The most important loop corrections are embodied in $\kappa(0)=1+\mathcal{O}(\alpha)$. They come from $\gamma-Z$ mixing and the anapole moment diagrams illustrated in fig. 2. They are normalized at $Q^{2}=0$. Effects due to $Q^{2} \neq 0$ are absorbed in $F_{2}\left(y, Q^{2}\right)$ which will be discussed later. Evaluated in a free field framework (i.e. ignoring strong interactions for the moment)

$$
\begin{aligned}
\kappa(0)= & -\frac{\alpha}{2 \pi s^{2}}\left\{\frac{1}{3} \sum_{f}\left(T_{3 f} Q_{f}-2 s^{2} Q_{f}^{2}\right) \ln \frac{m_{f}^{2}}{m_{Z}^{2}}\right. \\
& \left.-\left(\frac{7}{2} c^{2}+\frac{1}{12}\right) \ln c^{2}+\left(\frac{7}{9}-\frac{s^{2}}{3}\right)\right\}
\end{aligned}
$$

where $T_{3 f}= \pm 1 / 2$ (weak isospin) and $Q_{f}=$ fermion electric charge. The sum over all fermions (quarks and leptons) with mass $<m_{Z}$ comes from diagram 2a. (The top quark decouples completely from $\kappa(0)$ because of the specific definition of $\sin ^{2} \theta_{W}\left(m_{Z}\right)$ we are using [11].) The second and third terms stem from diagrams $2 \mathrm{~b}$ and $2 \mathrm{c}$ respectively.

The quark contributions in (14) cannot be properly accounted for perturbatively. Instead, one must use a dispersion relation to relate those vacuum polarization effects to $e^{+} e^{-} \rightarrow$ hadrons data. Such an analysis replaces the quark sum in (14) by [9, 12

$$
\frac{1}{3} \sum_{\text {quarks }}\left(T_{3 f} Q_{f}-2 s^{2} Q_{f}^{2}\right) \ln \frac{m_{f}^{2}}{m_{Z}^{2}} \rightarrow-6.88 \pm 0.50
$$

where the error assigned \pm 0.50 is rather conservative. We suspect that it would be lowered somewhat by an updated analysis of $e^{+} e^{-} \rightarrow$ hadrons data. Such a study may, one day, be 
important, since the error in (15) will turn out to be the dominant theoretical uncertainty and close to the projected experimental error presently attainable.

Numerically evaluating (14), one finds

$$
\kappa(0)=1.0301 \pm 0.0025
$$

That correction is very significant. It reduces the predicted $A_{L R}$ by about $38 \%$. The reason for that sensitivity is the fact that the quark loop diagrams in fig. 2 are not suppressed by $1-4 s^{2}$. Alternatively, one can say that $\kappa(0) \sin ^{2} \theta_{W}\left(m_{Z}\right)_{\overline{\mathrm{MS}}}$ is the effective low energy mixing angle appropriate for small $Q^{2} \sim 0$ rather than $\sin ^{2} \theta_{W}\left(m_{Z}\right)_{\overline{\mathrm{MS}}}$. The $3 \%$ increase due to the running of $\sin ^{2} \theta_{W}$ gets enhanced because of the $1-4 s^{2}$ sensitivity.

The next source of one loop corrections comes from the $W W$ and $Z Z$ box diagrams in fig. 3. The $W W$ box is not suppressed by $1-4 s^{2}$ and gives rise to the $\alpha\left(m_{Z}\right) / 4 \pi s^{2}$ term in (10). Taken alone that diagram gives a $4 \%$ enhancement of $A_{L R}$ relative to the lowest order prediction. The $Z Z$ box diagrams are suppressed by $1-4 s^{2}$. Hence, their contribution, the $3 \alpha\left(m_{Z}\right)\left(1-4 s^{2}\right)\left[1+\left(1-4 s^{2}\right)^{2}\right] / 32 \pi s^{2} c^{2}$ term in (10) is tiny, $\mathcal{O}(0.1 \%)$.

The next set of loops is illustrated in fig. 4. Together with photonic corrections to the external legs and vertices in fig. 1 and two photon exchange diagrams, they give rise to $Q^{2}$ dependent corrections denoted by $F_{1}\left(y, Q^{2}\right)$ in (10). We find

$$
\begin{aligned}
& F_{1}\left(y, Q^{2}\right)=-\frac{\alpha}{4 \pi}\left(1-4 s^{2}\right)\left\{\frac{22}{3} \ln \frac{y m_{Z}^{2}}{Q^{2}}+\frac{85}{9}+f(y)\right\}, \\
& f(y)=-\frac{2}{3} \ln [y(1-y)]+\frac{1}{\left(1-y+y^{2}\right)^{2}}\{ \\
& -2(1-y)\left(3-3 y+4 y^{3}-3 y^{4}\right) \ln (1-y) \\
& -2 y\left(1+3 y-6 y^{2}+8 y^{3}-3 y^{4}\right) \ln (y) \\
& +(1-y)\left(2-2 y-7 y^{2}+10 y^{3}-8 y^{4}+3 y^{5}\right) \ln ^{2}(1-y) \\
& -y\left(2-3 y-5 y^{2}+8 y^{3}-7 y^{4}+3 y^{5}\right) \ln ^{2}(y) \\
& +\left(2-4 y+11 y^{3}-13 y^{4}+9 y^{5}-3 y^{6}\right) \\
& \left.\times\left[\pi^{2}-2 \ln (1-y) \ln (y)\right]\right\}
\end{aligned}
$$

For the maximum asymmetry, $y=1 / 2$, one finds

$$
f\left(\frac{1}{2}\right)=\frac{17}{12} \pi^{2}+\frac{70}{9} \ln 2-\frac{8}{3} \ln ^{2} 2 \approx 18.09
$$

The actual evaluation of $F_{1}$ requires a value of $\sin ^{2} \theta_{W}$. Should we use $\sin ^{2} \theta_{W}\left(m_{Z}\right) \overline{\mathrm{MS}}=$ 0.2314 or $\kappa(0) \sin ^{2} \theta_{W}\left(m_{Z}\right) \overline{\mathrm{MS}}=0.2384$ in (17)? A proper treatment requires a renormalization group analysis of higher order leading logs. Instead of carrying out that study, we use the average of those two values and use their spread to estimate a theoretical uncertainty. In that way, we find for $\left\langle Q^{2}\right\rangle=0.02 \mathrm{GeV}^{2}$

$$
F_{1}\left(1 / 2,0.02 \mathrm{GeV}^{2}\right)=-0.0041 \pm 0.0010
$$


The final contribution that we need to consider is the effect of vacuum polarization in the $\gamma \gamma$ and $\gamma Z$ mixing self-energies for $Q^{2} \neq 0$. Because we have chosen to normalize $\alpha$ and $\kappa(0)$ at zero momentum transfer, there can be a correction for $Q^{2}$ non zero. Fortunately, the residual $Q^{2} \neq 0$ loop contributions largely cancel out (particularly for $y=1 / 2$ ). In terms of the $\gamma \gamma$ and $\gamma Z$ vacuum polarization function $\Pi_{\gamma \gamma}$ and $\Pi_{\gamma Z}$, one finds

$$
\begin{aligned}
& F_{2}\left(y, Q^{2}\right)= \\
& -4 c s\left[\frac{1}{2}\left(\Pi_{\gamma Z}\left(-Q^{2}\right)+\Pi_{\gamma Z}\left(-\frac{1-y}{y} Q^{2}\right)\right)-\Pi_{\gamma Z}(0)\right] \\
& +\left(1-4 s^{2}\right) \\
& \times\left[\frac{1}{2}\left(\Pi_{\gamma \gamma}\left(-Q^{2}\right)+\Pi_{\gamma \gamma}\left(-\frac{1-y}{y} Q^{2}\right)\right)-\Pi_{\gamma \gamma}(0)\right] \\
& -\left(1-4 s^{2}\right)\left(\frac{1}{2}-y\right) \frac{1+y(1-y)}{1-y(1-y)} \\
& \times\left[\Pi_{\gamma \gamma}\left(-\frac{1-y}{y} Q^{2}\right)-\Pi_{\gamma \gamma}\left(-Q^{2}\right)\right]
\end{aligned}
$$

For $y=1 / 2$, the last piece vanishes and lepton loops completely cancel. One finds

$$
\begin{aligned}
F_{2}\left(y=1 / 2, Q^{2}\right)= \\
-\left.4 c s\left[\Pi_{\gamma Z}\left(-Q^{2}\right)-\Pi_{\gamma Z}(0)\right]\right|_{\sin ^{2} \theta_{W}=1 / 4}
\end{aligned}
$$

where the partial cancellation of hadronic loops is simply accounted for by evaluating $\Pi_{\gamma Z}$, the vacuum polarization function, at $\sin ^{2} \theta_{W}=1 / 4$.

A proper evaluation of (21) requires a study of $e^{+} e^{-} \rightarrow$ hadrons data via dispersion relations similar to what went into (15). However, for $Q^{2}$ relatively small, one can approximate hadronic contributions to $\Pi_{\gamma Z}\left(-Q^{2}\right)-\Pi_{\gamma Z}(0)$ using a pion loop calculation. That rough approach gives

$$
\begin{aligned}
F_{2}\left(y=1 / 2, Q^{2}\right)_{\text {pions }} & \approx \frac{\alpha}{4 \pi}\left(\frac{A^{3}}{3} \ln \frac{A+1}{A-1}-\frac{2}{9}-\frac{2}{3} A^{2}\right) \\
A & \equiv\left(1+\frac{4 m_{\pi}^{2}}{Q^{2}}\right)^{1 / 2}
\end{aligned}
$$

For $Q^{2} \approx 0.025 \mathrm{GeV}^{2}$, the maximum at SLAC, one finds

$$
F_{2}\left(1 / 2,0.025 \mathrm{GeV}^{2}\right) \approx 2 \times 10^{-5}
$$

which is negligible. So, it seems, that for any foreseeable fixed target effort one can neglect $F_{2}$. It is in the noise. Of course, if $Q^{2} \gg m_{\pi}^{2}$, a careful evaluation of $F_{2}\left(y, Q^{2}\right)$ would have to be undertaken.

The last issue that must be addressed is the effect of bremsstrahlung on $A_{L R}$. We have not included that effect because it is dependent on the kinematic acceptance of a given 
experiment. However, we do expect on general grounds, that bremsstrahlung is relatively unimportant. Our reasoning is as follows: soft photon effects, including radiation damping, factorize and cancel in the asymmetry ratio. Hard bremsstrahlung should also largely cancel, although the degree of cancellation probably depends on details of the experimental geometry. What contribution remains is proportional to $\frac{\alpha}{\pi}\left(1-4 \kappa(0) \sin ^{2} \theta_{W}\left(m_{Z}\right) \overline{\mathrm{MS}}\right)$ and hence, likely to be very small. Therefore, neglect of bremsstrahlung, seems justified at the level of theoretical and experimental uncertainties we are considering. Of course, if a specific experiment is carried out, correcting for bremsstrahlung effects is straightforward and should be addressed by the experimentalists.

Collecting all of the one loop radiative corrections, one finds for $y=1 / 2$ and $Q^{2}=$ $0.025 \mathrm{GeV}^{2}$

$$
\begin{aligned}
1-4 \sin ^{2} \theta_{W} \rightarrow & 1.00122[1-4(1.0301 \pm 0.0025)(0.2314) \\
& +0.0027-0.0001-0.0041 \pm 0.0010]
\end{aligned}
$$

or

$$
0.0744 \rightarrow 0.0450 \pm 0.0023 \pm 0.0010
$$

That represents a $40 \pm 3 \%$ reduction in the asymmetry due to quantum loop effects. The reduction is rather insensitive to $y$ or $Q^{2}$ (unless we go to extreme values). That $40 \%$ reduction also (roughly) applies to the parity violating electron-electron interaction of interest in atomic parity violation [13]. (In fact, the reduction there is about 43\%.) It renders what was already a tiny effect essentially negligible.

For $Q^{2}=0.025 \mathrm{GeV}^{2}$ and $y=1 / 2$, as envisioned in a potential SLAC experiment, one finds that the radiative corrections reduce $A_{L R}\left(e^{-} e^{-}\right)$from $2.97 \times 10^{-7}$ to $(1.80 \pm 0.09 \pm$ $0.04) \times 10^{-7}$. The theoretical uncertainties in that result are roughly at the level of present experimental statistical capabilities. They are, however, somewhat conservative. One could imagine that further scrutiny of $e^{+} e^{-} \rightarrow$ hadrons data and use of the renormalization group to incorporate higher order leading logs could reduce the theoretical errors by about a factor of 2 . Hence, theory and realistic experimental precision are well matched.

A measurement of $A_{L R}\left(e^{-} e^{-}\right)$to $1.4 \times 10^{-8}$ may actually be made easier because of the reduction we have found. Indeed, some systematic uncertainties which depend on polarization monitoring uncertainties are proportional to $A_{L R}$ and hence also reduced by $40 \%$.

From our results, one sees that a determination of $A_{L R}$ to $\pm 1.4 \times 10^{-8}$ measures the standard model radiative corrections at about the 7 sigma or more level. Those corrections stem mainly from $\gamma Z$ vacuum polarization effects and can be viewed as the running of $\sin ^{2} \theta_{W}(\mu) \overline{\mathrm{MS}}$ from its value 0.2314 at $\mu=m_{Z}$ to a $3 \%$ larger value at $\mu=0$. Confirming that loop prediction of the standard model would certainly be an important result. Of course, such sensitivity implies that a measurement of $A_{L R}$ to $\pm 1.4 \times 10^{-8}$ is likely to also be a good probe of "new physics". We, therefore, now describe its potential for several examples of physics beyond the standard model. 


\section{III. "NEW PHYSICS" SENSITIVITY}

Comparison of a precise measurement of $A_{L R}$ with the standard model prediction can provide a sensitive probe of "new physics". It requires, of course, a "new physics" contribution to the parity violating $e^{-} e^{-} \rightarrow e^{-} e^{-}$amplitude. Also, $A_{L R}$ can indicate a deviation from the standard model, but cannot specify the source. Nevertheless, it is instructive to examine various "new physics" scenarios and compare their implications for $A_{L R}$ and other precision measurements. Here, we consider a few representative examples. For each case, we quote the $1 \sigma$ reach of $A_{L R}$, assuming a standard model central prediction of $1.8 \times 10^{-7}$ (for $y=1 / 2$ and $Q^{2}=0.025 \mathrm{GeV}^{2}$ ) and a total uncertainty (experimental and theoretical) of $\pm 1.4 \times 10^{-8}$, i.e. a $\pm 7.8 \%$ confrontation.

\section{A. $Z^{\prime}$ bosons}

Grand unified theories, such as $S O(10)$ and $E_{6}$, often predict the existence of additional neutral gauge bosons, collectively called $Z$ 's. The masses of those particles are not specified, but could under certain conditions be relatively light, $\mathcal{O}(1 \mathrm{TeV})$, and nevertheless beyond the reach of current experiments. For definiteness, we consider the $E_{6}$ model [12] which contains two $Z^{\prime}$ eigenstates (with $m_{Z_{\beta}}<m_{Z_{\beta}^{\prime}}$ )

$$
\begin{aligned}
Z_{\beta}= & Z_{\chi} \cos \beta+Z_{\psi} \sin \beta \\
Z_{\beta}^{\prime}= & -Z_{\chi} \sin \beta+Z_{\psi} \cos \beta \\
& -\frac{\pi}{2} \leq \beta \leq \frac{\pi}{2}
\end{aligned}
$$

$E_{6}$ symmetry specifies the couplings to electrons (up to some renormalization uncertainties) and one finds that $A_{L R}$ is increased by a factor [12]

$$
\begin{aligned}
1+7\left\{\frac{m_{Z}^{2}}{m_{Z_{\beta}}^{2}}\left(\cos ^{2} \beta+\sqrt{\frac{5}{3}} \sin \beta \cos \beta\right)\right. \\
\left.+\frac{m_{Z}^{2}}{m_{Z_{\beta}^{\prime}}^{2}}\left(\sin ^{2} \beta-\sqrt{\frac{5}{3}} \sin \beta \cos \beta\right)\right\}
\end{aligned}
$$

For an (effective) $S O(10)$ model, $\beta=0$, that expression simplifies to

$$
1+7 \frac{m_{Z}^{2}}{m_{Z_{\chi}}^{2}}
$$

Hence, at the $1 \sigma$ level, $m_{Z_{\chi}} \approx 870 \mathrm{GeV}$ is probed. That reach is roughly equivalent to a $\pm 1 \%$ determination of atomic parity violation in cesium [14] 16]. It is also comparable to the discovery reach of an upgraded Tevatron $p \bar{p}$ collider. 


\section{B. Electron Anapole Moment}

The electron matrix element of the electromagnetic current, $J_{\mu}^{\mathrm{em}}$, can be written as (with $\left.q=p^{\prime}-p\right)$

$$
\begin{aligned}
& \left\langle e\left(p^{\prime}\right)\left|J_{\mu}^{\mathrm{em}}\right| e(p)\right\rangle=\bar{u}_{e}\left(p^{\prime}\right) \Gamma_{\mu} u_{e}(p) \\
& \Gamma_{\mu}=F_{1}\left(q^{2}\right) \gamma_{\mu}+i F_{2}\left(q^{2}\right) \sigma_{\mu \nu} q^{\nu}-F_{3}\left(q^{2}\right) \sigma_{\mu \nu} q^{\nu} \gamma_{5} \\
& \quad+F_{A}\left(q^{2}\right)\left(\gamma_{\mu} q^{2}-2 m_{e} q_{\mu}\right) \gamma_{5}
\end{aligned}
$$

The first three form factors at $q^{2}=0$ give the electric charge, anomalous magnetic moment, and electric dipole moment (in units of $e$ ). All three are physical observables. The parity violating form factor $F_{A}\left(q^{2}\right)$ at $q^{2}=0$ is called the anapole moment. It is not a direct physical observable and suffers from electroweak gauge ambiguities. Indeed, in the standard model it is merely a part of the total loop corrections to a physical quantity and cannot be uniquely disentangled. Nevertheless, it is, in principle, possible that some forms of "new physics" contribute to $A_{L R}$ primarily through the electromagnetic anapole moment. Alternatively, one can view constraints on $F_{A}(0)$ as providing a figure of merit for comparing different measurements.

The anapole moment interaction in (29) would shift the $A_{L R}$ prediction by a factor

$$
\left(1+\frac{8 \sqrt{2} \pi \alpha}{G_{\mu}\left(1-4 \sin ^{2} \theta_{W}\right)} F_{A}(0)\right)
$$

or in units of the $W$ boson mass

$$
\left(1+77 m_{W}^{2} F_{A}(0)\right)
$$

Therefore, a measurement of $A_{L R}$ to $\pm 7.8 \%$ probes

$$
F_{A}(0)= \pm \frac{1 \times 10^{-3}}{m_{W}^{2}} \approx \pm\left(8 \times 10^{-18} \mathrm{~cm}\right)^{2}
$$

That level of sensitivity compares very favorably with other studies [17]. It corresponds to atomic parity violation in cesium at about the $\pm 0.3 \%$ level.

\section{The $X$ parameter}

If high mass scale "new physics" enters through gauge boson propagators, it is conveniently studied using the Peskin-Takeuchi $S, T$, and $U$ parameters 18. If the scale of the "new physics" is $\mathcal{O}\left(m_{Z}\right)$, rather than $\gg m_{Z}$, that formalism should be extended to $S, T$, $U, V, W, X\left[19,20\right.$. The additional quantities parametrize changes from $Q^{2} \approx 0$ to $m_{Z}^{2}$ due to "new physics" loops. In that approach, our $\kappa(0)$ in eq. (14) gets multiplied by [20]

$$
(1-0.032 X)
$$

A measurement of $A_{L R}$ to $\pm 7.8 \%$ or $\Delta \sin ^{2} \theta_{W}$ to \pm 0.0011 then constrains $X$ at the \pm 0.14 level. That is to be compared with global fits to all existing data 20] which currently give $X=0.38 \pm 0.59$. So, an $A_{L R}$ measurement could improve the constraint by a factor of 4 or so. 


\section{Generic Loops}

If we parametrize "new physics" loop contributions to $A_{L R}$ by a general parity violating 4 Fermi interaction

$$
C \frac{\alpha^{2}}{M^{2}} \bar{e} \gamma_{\mu} \gamma_{5} e \bar{e} \gamma^{\mu} e
$$

with $M$ (roughly) the "new physics" mass scale, it modifies $A_{L R}$ by a factor

$$
\left(1+0.05 C \frac{m_{W}^{2}}{M^{2}}\right)
$$

In theories with $C \approx 1$, we see that a $\pm 7.8 \%$ measurement of $A_{L R}$ explores the $M \approx m_{W}$ scale. That is in keeping with our finding that the $W W$ box diagram shifts $A_{L R}$ by about $+7 \%$. Of course, there can be enhancements or suppressions in the case of "new physics". It would be interesting to compute $C / M^{2}$ in classes of low mass supersymmetry models. That exercise is, however, beyond the scope of this paper.

\section{CONCLUSION}

We have calculated the one loop electroweak radiative corrections to the parity violating electron-electron interactions and found a rather substantial $40 \pm 3 \%$ reduction of the tree level prediction. That result further reduces (the already insignificant) role of the electron-electron interaction in atomic parity violation and has interesting consequences for the left-right asymmetry in polarized Møller scattering. It is clear that any future precision

measurement of $A_{L R}$ must be cognizant of those large corrections. We also showed that an experimental determination of $A_{L R}$ at the $\pm 7.8 \%$ level provides a useful and competitive probe of "new physics". Used in conjunction with other precision measurements and direct high energy probes it may unveil and help to decipher physics beyond the standard model.

\section{ACKNOWLEDGMENTS}

A.C. would like to thank Professor W. Hollik for many discussions and advice. A.C.'s research was supported by BMFT $056 \mathrm{KA}$ 93P. W.J.M. would like to thank the Institute for Nuclear Theory for its hospitality during the completion of this work. This manuscript has been authored under contract number DE-AC02-76CH00016 with the U.S. Department of Energy. Accordingly, the U.S. Government retains a non-exclusive, royalty-free license to publish or reproduce the published form of this contribution, or allow others to do so, for U.S. Government purposes. 


\section{REFERENCES}

[1] C. Prescott et al., Phys. Lett. B77, 347 (1978).

[2] P. A. Souder et al., Phys. Rev. Lett. 65, 694 (1990).

[3] E. Derman and W. J. Marciano, Ann. Phys. 121, 147 (1979).

[4] K. Kumar, E. Hughes, R. Holmes, and P. Souder, Parity Nonconservation in Møller Scattering, Princeton preprint, 1995.

[5] R. J. Finkelstein, R. E. Behrends, and A. Sirlin, Phys. Rev. 101, 866 (1956).

[6] T. Kinoshita and A. Sirlin, Phys. Rev. 113, 1652 (1959).

[7] A. Sirlin, Phys. Rev. D22, 971 (1980).

[8] W. J. Marciano and A. Sirlin, Phys. Rev. D22, 2695 (1980).

[9] W. J. Marciano and A. Sirlin, Phys. Rev. D27, 552 (1983).

[10] W. J. Marciano and A. Sirlin, Phys. Rev. Lett. 46, 163 (1981).

[11] P. Gambino and A. Sirlin, Phys. Rev. D49, R1160 (1994).

[12] W. J. Marciano, in Proceedings of the 1993 SLAC Summer Institute "Spin Structure in High Energy Processes", SLAC-Report-444.

[13] S. Blundell, J. Sapirstein, and W. Johnson, Phys. Rev. D45, 1602 (1992).

[14] M. C. Noecker, B. P. Masterson, and C. E. Wieman, Phys. Rev. Lett. 61, 310 (1988).

[15] D. London and J. Rosner, Phys. Rev. D34, 1530 (1986).

[16] W. J. Marciano and J. Rosner, Phys. Rev. Lett. 65, 2963 (1990).

[17] G. Kopp, D. Schaile, M. Spira, and P. Zerwas, Z. Phys. C65, 545 (1995).

[18] M. Peskin and T. Takeuchi, Phys. Rev. Lett. 65, 964 (1990).

[19] I. Maksymuk, C. P. Burgess, and D. London, Phys. Rev. D50, 529 (1994).

[20] A. Kundu and P. Roy, preprint SINP-TNP-94-07, hep-ph/9411225. 


\section{FIGURES}

FIG. 1. Neutral current amplitudes leading to the asymmetry $A_{L R}$ at tree level.

FIG. 2. $\quad \gamma-Z$ mixing diagrams (a-b) $W$-loop contribution to the anapole moment (c).

FIG. 3. Box diagrams with two heavy bosons.

FIG. 4. Boxes containing one photon and $Z$-loop contribution to the anapole moment. 